\title{
Optimizing Stand Spatial Structure by Using Neighborhood-Based Quantitative Indicators: A Case of Boreal Forests
}

\author{
Lingbo Dong \\ Northeast Forestry University https://orcid.org/0000-0003-0079-2688 \\ Pete Bettinger \\ University of Georgia \\ Zhaogang Liu ( $\sim$ lzg19700602@163.com ) \\ Northeast Forestry University
}

\section{Research}

Keywords: stand structure, boreal forests, species mingling, combinatorial optimization, neighborhoodbased indicator

Posted Date: October 11th, 2021

DOI: https://doi.org/10.21203/rs.3.rs-940208/v1

License: (c) (i) This work is licensed under a Creative Commons Attribution 4.0 International License. Read Full License 

quantitative indicators: a case of boreal forests

${ }^{a}$ Key laboratory of Sustainable Forest Ecosystem Management-Ministry of Education, College of Forestry, Northeast Forestry

${ }^{\mathrm{b}}$ Warnell School of Forestry and Natural Resources, University of Georgia, Athens 30602 GA, USA; pbettinger@ warnell.uga.edu;

* Corresponding author: lzg19700602@163.com (Z. Liu)

\section{Abstract:}

Background: Over the past fifty years, societies have placed increasing demands on forests, and their use has shifted gradually from wood production to the diversified benefits and functions of ecosystem services. The effects of neighborhood-based structural characteristics on regulating growth and promoting sustainability have therefore drawn much attention. However, direction for managing natural mixed forests using neighborhood-based indicators are still not clear.

Methods: In this study, a tree-level harvest planning tool that considers four neighborhood-based structural parameters (species mingling, diametric differentiation, horizontal spatial pattern and crowdedness of trees) while concurrently recognizing other operational constraints, was developed using simulated annealing algorithm. The approach was applied to four 1-ha mapped stands in northeast China, namely a natural larch forest (NLF), a natural birch forest (NBF), a natural secondary forest (SEF), and a Korean pine broad-leaved forest (KBF). 
Results: The tree-level harvest optimization improved the objective function values by approximately $78.33 \%$ of NLF, and $134.96 \%$ of NBF, and $156.70 \%$ of SEF and $252.95 \%$, respectively. The optimal harvest intensities for partial cutting activities varied from $22.16 \%$ (SEF) to $26.07 \%$ (NBF) of the standing volume. In evaluating the four neighborhood-based structural parameters, both species mingling and crowdedness have the highest priority to be used in structure-based forest management.

Conclusions: Our results demonstrated that that the commonly used neighborhood-based structural parameters could be used to control the spatial layout of potential harvest trees, in turn may be conducive to regulate the growth and stability of forests.

Keywords: stand structure; boreal forests; species mingling; combinatorial optimization; neighborhood-based indicator

\section{Background}

Forest structures are the bases of architectural and functional of forest ecosystems, which play an important role in regulating the regeneration, growth, and sustainability of natural mixed uneven-aged forests (Chanthorn et al., 2017). Commonly used forest structural indices can be divided into two groups depending on whether spatial information (the location of trees) is needed. The non-spatial group focuses mainly on the quantities and qualities of tree characteristics (e.g., diameter distribution and species composition) that formulate the basis of forest communities. The spatial group focuses on aspects that mainly refer to the locations of trees and the spatial associations amongst them (Hui and Gadow, 2003; Pastorella and Paletto, 2013). These spatial aspects can not only determine the intensity of competitive between neighboring trees, but also the spatial niche found between trees and the growth potential and stability of the surrounding forest (Hui and Gadow, 2003; Gao et al, 2021). Therefore, the spatial aspects regarding the location of individual trees has often been recognized as more important than the non-spatial aspects (Dong et al., 2020). 
A set of tree-level spatial structural measurements have been proposed as important and reflective of the nearest neighbor relations between a reference tree and its $n$ nearest neighbor trees (called a "structural unit") (Figure 1; Hui, 2003; Davies and Pommerening, 2008; Li et al., 2014; Dong et al., 2020). These measurements include the uniform angle $(W)$, mingling index $(M)$, dominance index $(D)$, and crowdedness index $(C)$. The measurements of spatial relationships reflect the distribution, diversity, size variation, juxtaposition of trees, respectively, within a unit of area (Hui and Gadow, 2003). The selection of neighbouring trees (e.g., how many neighbors) is a critical issue in the measurement of stand spatial structure (Wang et al., 2016). Methods have been devised to arrive at the reasonable number of neighbouring trees, such as nearest neighbour, fixed radii and Voronoi tessellations (Pommerening and Stoyan, 2006). However, Hui and Gadow (2003) and Wang et al. (2016) both indicated that four neighbours were enough for these measurements, when one considers sampling accuracy and costs simultaneously. Expanding from the tree-level to the stand-level, average values of these measures can help explain the structural differences between stands composed of different forest types, positioned at different successional stages, and influenced by different management activities (e.g., Wan et al., 2019).

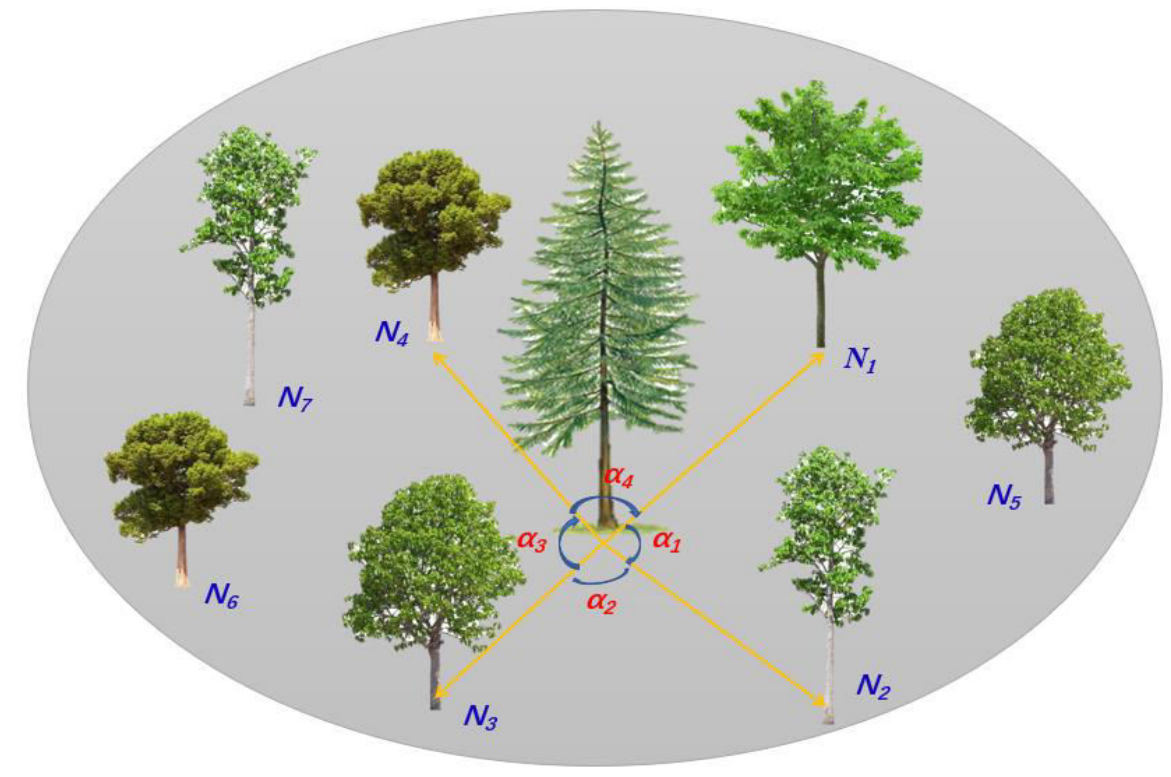

59 Figure 1 Schematic diagram of a structural unit, in which $N_{l} \sim N_{4}$ are the four nearest neighbours of a reference tree, $N_{5} \sim N_{6}$ are also close to the reference tree but are not the nearest neighbours, $\alpha_{1} \sim \alpha_{4}$ are the angles between 
neighbouring trees with the reference tree positioned as the angle corner.

Due to a variety of anthropogenic disturbances (e.g., timber harvesting), the structure and function of natural forests around the world have been altered (State Forestry Bureau, 2016). Methods for accelerating the recovery process and improving the quality of natural forests is a current topic of conversation in the field of forest research. Historically, several management strategies, such as the comprehensive indices approach (Gadow and Hui, 1999), the stem number guide curve (Newton, 2012; Peng et al., 2018), the target tree-oriented management approach (Song, 2015), and the value growth rate method (Martín-Fernández and García-Abril, 2005; Packalen et al., 2020), have been assessed for their value in accelerating the recovery process and improving the quality of natural forests. However the speed of restoration and the resulting structural complexity usually found to be not as favorable as expected for natural mixed forests (Wan et al., 2019; Díaz-Yáez et al., 2019). The key reason for this phenomenon may be that most management strategies focus only on wood production, rather than structural features of forest ecosystems. There are exceptions, such as the stand-level optimization approach presented in Bettinger et al. (2005) that attempted to maximize stand density over time by assessing contributions of individual living trees.

Recently, some studies have suggested that certain stand spatial structures could act to significantly improve the growth of individual trees (Davies and Pommerening, 2008; Pommerening and Uria-Diez, 2017; Lyu et al., 2020). Thus, for the purposes of improving forest health and projected outcomes from the management of natural forests, integrating tree-level structural indices in the harvest selection process has drawn much attention. For instance, Pastorella and Paletto (2013), Ye et al. (2018) and Dong et al. (2020) all simulated selective thinning processes using a comprehensive index method that was based on frequently used spatial and non-spatial structure indices. Li et al. (2017) optimized the structure of a stand using bivariate probability distributions of three spatial parameters (i.e., $U$-, $M$-, and $D$-indices). However, all of these works ignore an important issue regarding tempoal 
changes in spatial structure measurements: the neighbors of a reference tree may dynamically change as thinning processes are scheduled. For example, N1-N4 are the four nearest neighbors of the current reference tree (Figure 1), however the structural unit around the reference tree may become as N2, N3, N4 and N7 if tree N1 is assigned for harvest. When thinnings or selective harvests are considered, it is logically impossible to know every potential structural unit a priori. Thus, utilizing optimization or simulation processes to adjust the structural unit around each tree when harvests are scheduled can assist in understanding the impact of harvests on ecological systems. However, during our literature review, we found that Bettinger and Tang (2015) was the only tree-level harvest optimization process that mainly focused on improving the species mingling of natural forests. However, they did not consider the other spatial aspects (e.g., size differentiation, spatial pattern and crown crowdedness), which have been recognized as important as local species diversity.

The main objective of this study is to develop a tree-level harvest planning tool for natural mixed forests in northeast China. For this purpose, (1) we firstly develop a tree-level planning formulation based on optimizing four commonly used neighborhood-based structural parameters (i.e., $M, W, D, C$ ) that was subject to a set of thinning constraints, (2) we try to solve the nonlinear integer programming problem using a $s$-metaheuristic (simulated annealing) algorithm since prior knowledge of the structural unit for each tree is difficult to know aforehand, and (3) we apply the tree-level harvest planning model to four different natural mixed forests from northeast China.

\section{Methods}

Study area and data

As case studies, data for four different natural mixed forests were collected from within the Heilongjiang Province in Northeast China (Figure 2). The first two forests were composed of natural larch (Larix gmelinii) (NLF) and natural birch (Betula platyphylla) (NBF). These were located in the central part of Great Xing'an Mountain 
105

106

107

108

109

110

$\left(123^{\circ} 20^{\prime}-124^{\circ} 21^{\prime} \mathrm{E}, 52^{\circ} 16^{\prime}-52^{\circ} 47^{\prime} \mathrm{N}\right)$ in the cold-temperate zone, where the mean annual air temperature is $-2.8^{\circ} \mathrm{C}$ and the mean annual precipitation level is about $450 \mathrm{~mm}$. Precipitation events occurs primarily in summer in this part of the Heilongjiang Province. Due to the higher latitude of this location, as compared to the other case studies, species abundance is relatively lower. Here, larch and birch species usually coexist in NLF forests, while birch is usually most frequent tree species in NBF forests. Other tree species that may be found in these forest types include spruce (Picea asperata), Scots pine (Pinus sylvestris), and poplar (Populus davidiana).

The third forest is composed of Korean pine (Pinus koraiensis) and other broadleaved tree species (KBF). This type of forest is reflective of the historical climax community of the Xiaoxing'an Mountains $\left(129^{\circ} 11^{\prime}-129^{\circ} 26^{\prime} \mathrm{E}, 46^{\circ} 32^{\prime}-46^{\circ} 39^{\prime} \mathrm{N}\right)$. The forest is situated in an area that has a temperate continental monsoon climate, where the mean annual air temperature is $2^{\circ} \mathrm{C}$, and the mean annual precipitation level is $600 \mathrm{~mm}$. The research plot that was measured in the third forest has been subjected to different intensities of commercial thinning activities. These were conducted in the 1990s by local communities for the purpose of generating income, however this forest has been developing naturally since. The vegetation in the research plot consists of more than 20 tree species that are native Xiaoxing'an Mountain flora. The main species are Korean pine (P. koraiensis), oak (Quercus mongolica), birch (B. platyphylla), other hardwoods (e.g., Fraxinus mandshurica, Phellodendron amurense, Juglans mandshurica, and Ulmus pumila) and softwood species (e.g., P. davidiana).

The fourth forest is representative of a naturally regenerated second growth forest (SEF), and is situated in a low-altitude area of the Zhangguangcai Mountains $\left(127^{\circ} 18^{\prime}-127^{\circ} 41^{\prime} \mathrm{E}, 45^{\circ} 2^{\prime}-45^{\circ} 18^{\prime} \mathrm{N}\right)$. The climate here is temperate, with a mean annual air temperature of $3.1^{\circ} \mathrm{C}$ and a mean annual precipitation level of $700 \mathrm{~mm}$. Following a clearfelling activity in 1920s, the research site was naturally colonised by trees; few management activities have occurred here since regeneration began. However, to promote regeneration in forest gaps, Korean pine seedlings were planted in 2004 at a density of approximately 500 trees per hectare. Species abundance in this 


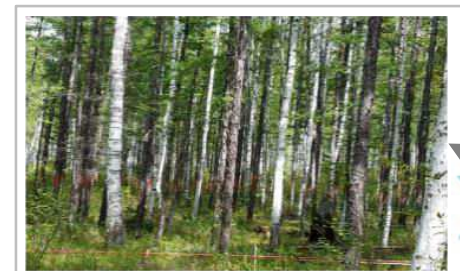

Natural larch forest (NLF)
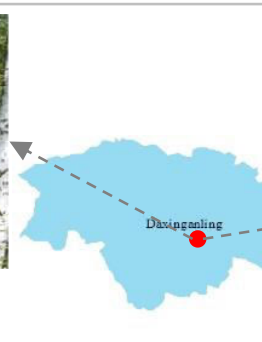
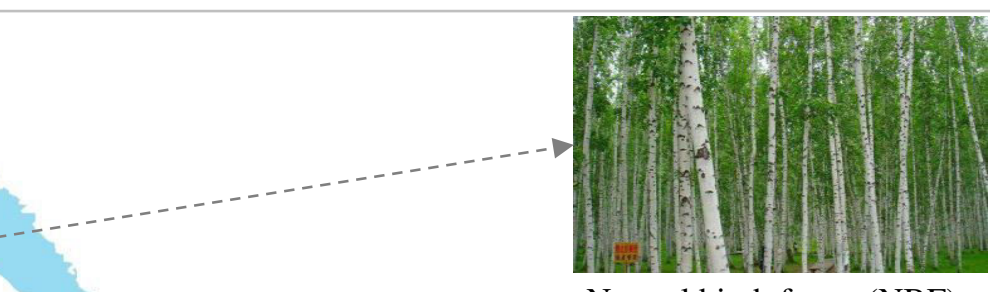

Natural birch forest (NBF)

Second growth forests (SEF)
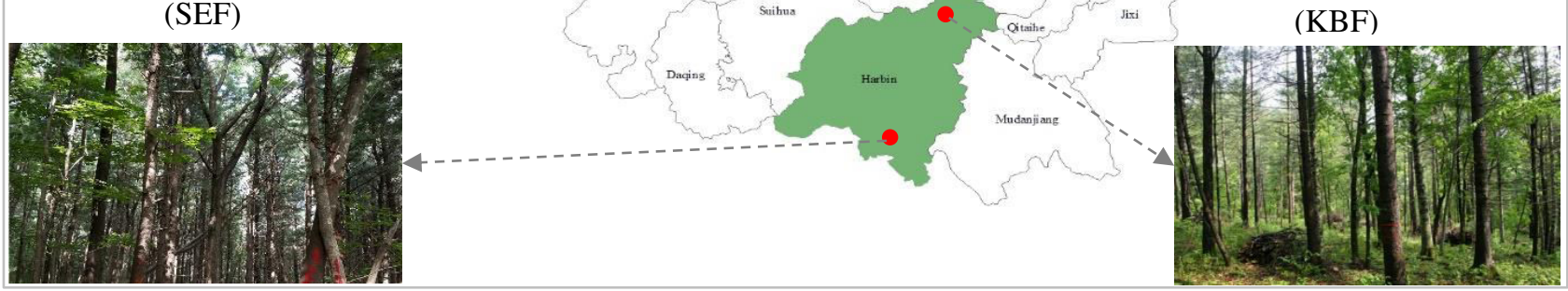

Figure 2 Location of the four studied forests in northeast China.

To conduct the optimization simulation, a 1-ha $(100 \mathrm{~m} \times 100 \mathrm{~m})$ permanent plot was established within each of the case study forests in the summer of 2016-2017. All trees with a diameter at breast height (DBH) $\geq 5 \mathrm{~cm}$ were marked, and their locations, species, DBH, total tree height (HT), crown width $(\mathrm{CW})$ in four directions (i.e., east, west, south and north), living branch height, and status (e.g., survival, diseases or insects) were recorded. The four case study areas were composed of forests that had tree densitities ranging from about 800 to 1400 trees per hectare, average diameters ranging from 10.5 to $15.8 \mathrm{~cm}$, and tree heights ranging from about 10 to $13 \mathrm{~m}$ (Table $1)$.

Table 1 Basic statistical characteristics of the four studied forests in northeast China

$\begin{array}{lccccccc} & \text { Altitude } & & \text { Slope } & \text { Mean DBH } & \text { Mean HT } & \text { Mean CW } & \text { Density } \\ \text { Forest types } & & \text { Aspect } & & & & \\ & (\mathrm{m}) & & \left(^{\circ}\right) & (\mathrm{cm}) & (\mathrm{m}) & \text { (m) } & \left(\text { trees }^{\circ} \mathrm{ha}^{-1}\right)\end{array}$




\begin{tabular}{|c|c|c|c|c|c|c|c|}
\hline Natural larch forest (NLF) & 546 & None & $<5$ & 10.54 & 10.09 & 1.30 & 1380 \\
\hline Natural birch forest (NBF) & 565 & None & $<5$ & 12.73 & 10.94 & 1.37 & 898 \\
\hline Korean pine broadleaved forest (KBF) & 399 & None & $<5$ & 15.78 & 13.15 & 2.23 & 813 \\
\hline Second growth forests (SEF) & 339 & North & 13 & 13.09 & 12.16 & 1.74 & 1206 \\
\hline
\end{tabular}

\section{Spatial structure parameters}

The spatial structural measurements for each structural unit (each reference tree, or averaged to the entire stand) were quantified using four different tree-level indices (Figure 1). These measurements included the mingling index $(M)$, the diameter dominance index $(D)$, the uniform angle index $(W)$ and the crowdedness index $(C$; Hui and Gadow, 2003). The mingling index describes the species composition and spatial pattern in multispecies forest, in which are usually defined as the proportion of $n$ nearest neighbours that are a different species from each reference tree. The dominance index characterizes the size (e.g., diameter, height) differentiations between each reference tree and its $n$ nearest neighbours, which can reflect the competition status between the reference tree and its $n$ nearest neighbours. The uniform angle index is used to measure the regularity degree of the $n$ nearest neighbours around each reference tree. The crowdedness index reflects the overlapping between the canopy of each reference tree and its $n$ nearest neighbours. The formulations of the four indices were stated in Dong et al. (2020) as: 
where $M_{i}, D_{i}, W_{i}$ and $C_{i}$ are the values of mingling degree, diameter dominance, uniform angle, and crowdedness for reference tree $i$, respectively; $n$ is the number of neighbours of reference tree $i$, where $n=4$ in this analysis; and $v_{i j}, k_{i j}, z_{i j}$ and $y_{i j}$ are discrete variables. Further, $s p_{i}$ and $s p_{j}$ represent the tree species of reference tree $i$ and neighbour tree $j$, respectively; $d b h_{i}$ and $d b h_{j}$ represent the $\mathrm{DBH}$ of reference tree $i$ and its neighbour tree $j$, respectively; and $L_{i j}$ represents the Euclidean distance between the boles of reference tree $i$ and its neighbour tree $j$. The following logic was employed in equations 1-4:

- If neighbour tree $j$ is not the same tree species as reference tree $i, v_{i j}=1$, otherwise $v_{i j}=0$

- If the DBH of neighbour tree $j$ is smaller than the DBH of reference tree $i, k_{i j}=1$, otherwise $k_{i j}=0$

- If the angle $\alpha$ of two neighbour trees is smaller than the expected standard angle $\alpha_{0}, z_{i j}=1$, otherwise $z_{i j}=0$

- If the sum of the crown width of neighbour tree $j\left(C W_{j}\right)$ and reference tree $i\left(C W_{i}\right)$ is less than the Euclidean distance between the boles of neighbour tree $j$ and reference tree $i\left(L_{i j}\right), y_{i j}=1$, otherwise $y_{i j}=0$

Within a structural unit (Figure 1), the four neighborhood-based structural measurements have five possible values: $0.00,0.25,0.50,0.75$, and 1.00 . These values represent different ecological conditions around a reference tree (Table 2). The standard angle $\left(\alpha_{0}\right)$ for the uniform angle index should be $\alpha_{0}=360^{\circ} / n$ or $90^{\circ}$ theoretically. However, this situation is very rare in nature, and at most only three of the four angles in a structural unit can be larger than $90^{\circ}$. Thus, following the suggestions of Pommerening and Stoyan (2006) and Hui et al. (2007), the standard angle was assumed to be $\alpha_{0}=360^{\circ} /(n+1)$, or $72^{\circ}$ in this analysis. For a specific structural unit (or a stand), the $W$ values in the interval of $[0.475,0.517]$ usually implies a random distribution pattern of trees, while $W<$ 0.475 would imply a regular pattern, and $W>0.517$ would imply a clumped pattern of trees. Obviously, $W$ values close to 0 indicate a low species mingling, a dominant growth status of the reference tree, a very regular 
179 horizontal distribution pattern, and a very sparse growth space. High $W$ values are suggestive of higher species 180 diversity, a disadvantaged growth status, clumped tree patterns, and a dense growth space. To avoid the edge 181 effects (Pommerening and Stoyan, 2006), trees located within the core area of each study site were considered as 182 either reference trees or neighbour trees, while trees located around the outer edges (with $5 \mathrm{~m}$ of the boundary) of 183 each study site were only considered to be neighbour trees.

Table 2 Classifications and ecological conditions around a reference tree when using a five-tree structural unit

\begin{tabular}{|c|c|c|c|c|c|c|}
\hline \multirow{2}{*}{ Index } & \multirow{2}{*}{ Variable } & \multicolumn{5}{|c|}{ Parameter values } \\
\hline & & 0.00 & 0.25 & 0.50 & 0.75 & 1.00 \\
\hline \multicolumn{7}{|l|}{ Mingling degree } \\
\hline & species & Non mixture & Low mixture & Intermediate mixture & High mixture & Complete mixture \\
\hline \multicolumn{7}{|l|}{$M \in[0,1]$} \\
\hline \multicolumn{7}{|l|}{ Dominance index } \\
\hline & diameter & Predominant & Subdominant & Intermediate & Disadvantaged & Absolutely disadvantaged \\
\hline \multicolumn{7}{|l|}{$D \in[0,1]$} \\
\hline \multicolumn{7}{|l|}{ Uniform angle index } \\
\hline & angle & Very regular & Regular & Random & Clumped & Very clumped \\
\hline \multicolumn{7}{|l|}{$W \in[0,1]$} \\
\hline \multirow[t]{2}{*}{ Crowdedness index } & distance & & & & & \\
\hline & & Very sparse & Sparse & Intermediate density & Dense & Very dense \\
\hline$C \in[0,1]$ & crown width & & & & & \\
\hline
\end{tabular}

As was suggested in section 2.2, the ecological complexity and character of a forest usually increases with 
191

neighborhood-based structural measurements simultaneously.

$$
z=\frac{\bar{M}}{\sigma_{M}} \cdot \frac{(1-\bar{C})}{\sigma_{C}} \cdot \frac{(1-\bar{D})}{\sigma_{D}} \cdot \frac{(1-|\bar{W}-0.5|)}{\sigma_{W}}
$$

subject to

$q_{l} \leq q \leq q_{u}$
$D C_{N}=D C_{0}$
$S_{N}=S_{0}$
$\bar{M}>\bar{M}_{0}$
$\bar{C}<\bar{C}_{0}$
$\bar{D}<\bar{D}_{0}$
$|\bar{W}-0.5|<0.015$
$\alpha_{l} N \leq N u m\left(x_{i}\right) \leq \alpha_{u} N$
$x_{i} \in[0,1]$

where $\mathrm{z}$ is the objective function value; $\bar{M}, \bar{C}, \bar{D}$ and $\bar{W}$ are the mean values of mingling degree, crowdedness index, diameter dominance index, and uniform angle index of the entire study area after thinning, respectively; $\sigma_{M}, \sigma_{C}, \sigma_{D}$ and $\sigma_{W}$ are the standard deviations of mingling degree, crowdedness index, diameter dominance index, and uniform angle index; $D C_{N}$ and $S_{N}$ are the number of diameter classes and tree species after thinning, while $D C_{0}$ and $S_{0}$ are the number of diameter classes and tree species before thinning; $N$ is the total number of trees within the plots; $\operatorname{Num}()$ is a counting function that used to sum the number of assigned harvest trees; $\alpha_{l}$ and $\alpha_{u}$ are the lower and upper limits for the harvest intensities that were qualified using the proportion of assigned harvest trees; $x_{i}$ is a binary variable representing the tree continuing to be alive $(0)$ or being harvested (1); $q$ is a measurement indicator that used to qualify the diameter distribution, and is calculated as $q=\exp (a \cdot d) ; a$ is the estimated parameter between the number of trees and diameter class when using the negative exponential function, 
namely $N=k \exp (-a \cdot d) ; d$ is the width of diameter class, where $d=2 \mathrm{~cm}$ in this analysis; $q_{l}$ and $q_{u}$ are the lower and upper limits of reasonable range of $q$-values in natural uneven aged forests.

Equation 5 represents the objective function of the planning problem, namely, to maximize the reasonableness of neighborhood-based structural characteristics at the stand-level. This is a unitless value described as the product of the four stand-level average neighborhood-based structural measurements after dividing each by their respective standard deviation. Equation 6 requires the $q$-value of diameter distribution after thinning should fall within the interval of $\left[q_{l}, q_{u}\right]$. Numerous previous studies (e.g., Schwartz et al., 2005; Kang 2010; Podlaski, 2017) have indicated that the reasonable ranges of $q$-value for natural uneven-aged forests usually varied between 1.2 and 1.7. Thus, $q_{l}$ and $q_{u}$ were respectively set as 1.2 and 1.7 in this analysis. Equations 7 and 8 constrain the number of tree species and diameter classes after thinning so that they are not less than that before thinning. Equation 9 indicates the optimization process should increase the stand mingling degree, while simultaneously decreasing the stand crowdedness index (Equation 10) and diameter dominance index (Equation11) simultaneously. Equation 12 requires the differences between $\bar{W}$-values after thinning and an expected value $(\bar{W}=0.5)$ to be less than 0.015 , namely maintaining the random distribution pattern of trees in horizontal dimension. Equation 13 limits the proportion of assigned harvest trees to a range from $\alpha_{l}$ to $\alpha_{u}$. According to recent published works (Macdonald et al., 2004; Li et al., 2014; Dong et al., 2020) and management practices (State Forestry Bureau, 2016), $\alpha_{l}$ and $\alpha_{u}$ are assumed to be $10 \%$ and $40 \%$, respectively. Equation 14 requires that the decision variables are binary.

\subsection{Simulated annealing algorithm}

Since the objective function and constraints are all computed and evaluated in a post-harvest manner, the neighbors of each reference tree may always be in a state of change (Figure 1), therefore it would be computationally burdonsome to know every potential structural unit a priori. Thus, the optimization procedures 
should dynamically obtain the correct combination of neighboring trees to construct the potential structural unit prior to solving the model. Thus, a s-metaheuristic algorithm (simulated annealing) was employed to solve the non-linear integer planning problem, rather than an exact method (mixed integer programming) where these potential relationships need to be known and listed in the problem formulation.

Simulated annealing (SA) was initially described by Kirkpatrick et al. (1983) although its foundations can be traced back several decades earlier (Metropolis et al., 1953). As a heuristic methods for solving combinatoiral forest planning problems, SA has been shown to be able to produce results very close to optimal (e.g., mixed integer programming) solutions in a number of forestry-based planning problems (Bettinger et al., 2002; Dong et al., 2018). In this heuristic, a move represents the random selection of a tree, and a change to the harvest decision: if currently scheduled for harvest it is unscheduled, if currently not scheduled for harvest it is scheduled. To avoid converging too slowly, and to avoid becoming stuck in an area of local optimality, selected moves that improve the objective function value are always accepted. Potential non-improving moves can also be accepted if a random number drawn from the computer is less than probability of acceptance $p=\operatorname{Exp}\left(-\left(z_{\text {new }}-z_{\text {old }}\right) / T\right)$. Here, $z_{\text {new }}$ and $z_{\text {old }}$ are objective function values for the current (potentally new) and previous (old) solution, and $T$ is the current temperature of the SA algorithm. During the search process, the temperature cools gradually $(T$ declines in value) according to a given cooling rate, which acts to decrease the probability of accepting non-improving moves as the search progresses. Near the end of the heuristic search process, $T$ is so low that the probability of acceptance for non-improving moves is nearly 0 , and the process acts as a hill-climbing heuristic.

In our implementation of SA, the search process is initially guided by a user-defined harvesting intensity (i.e., $30 \%$ of the trees) from which a feasible solution (i.e., a set of assigned harvest trees) would be generated randomly. From this point, three alternative moves could be made to diversify the potential harvesting intensities. The first strategy randomly schedules a previously unscheduled tree to the current harvest plan, which will increase the 
harvest intensity. These second strategy randomly unschedules a previously scheduled tree from the current harvest plan, which will decrease the harvest intensity. For the third strategy, a tree previously scheduled for harvest would be randomly replaced by a previously unscheduled selected tree, which keeps the harvest intensity consistent. The selection of these three strategies during a run of the SA algorithm was also random.

The tree-level harvest planning formulations and simulated annealing algorithm were programmed in $\mathrm{R}$ environment (R Core Team, 2021). The procedures was run on a $2.6 \mathrm{GHz}$ Core i5 processor with 4GB RAM and Windows 7 operating system. Parameters that were used to control the search processes of SA include the starting temperature, stopping temperature, cooling rate and the number of iterations at each temperature. Based on a set of trial-and-error tests, the values were set as 100, 1, 0.999 and 10, respectively. These parameters imply that each independent run will result in approximately 46031 potential iterations (assessments of potential moves). Ten solutions were generated for each forest and only the best solution (as identified as having the highest objective function value) was employed to perform an analysis of differences amongst the case study forests.

\section{Results}

The SA algorithm produced results that indicate the objective function values of each type of forest increased significantly with the increases of iterations (Figure 3), in which the increment proportions after thinning were approximately $78.33 \%$ of NLF, and $134.96 \%$ of NBF, and $156.70 \%$ of SEF and $252.95 \%$ of KBF when compared that with the statistics before thinning. The developments on the assigned harvest intensities of removing trees all decreased initially, however converged on their steady states finally. The optimal harvest intensities of the assigned removing trees were all close to $25 \%$, where the intensities for NBF (27.70\%) and SEF (27.35\%) were significantly larger than that of NLF (24.96\%) and KBF (24.85\%). The corresponding harvest intensities of removing volumes were respectively $24.49 \%$ of NLF, and $26.07 \%$ of NBF, and $22.16 \%$ of SEF and $22.23 \%$ of KBF. 

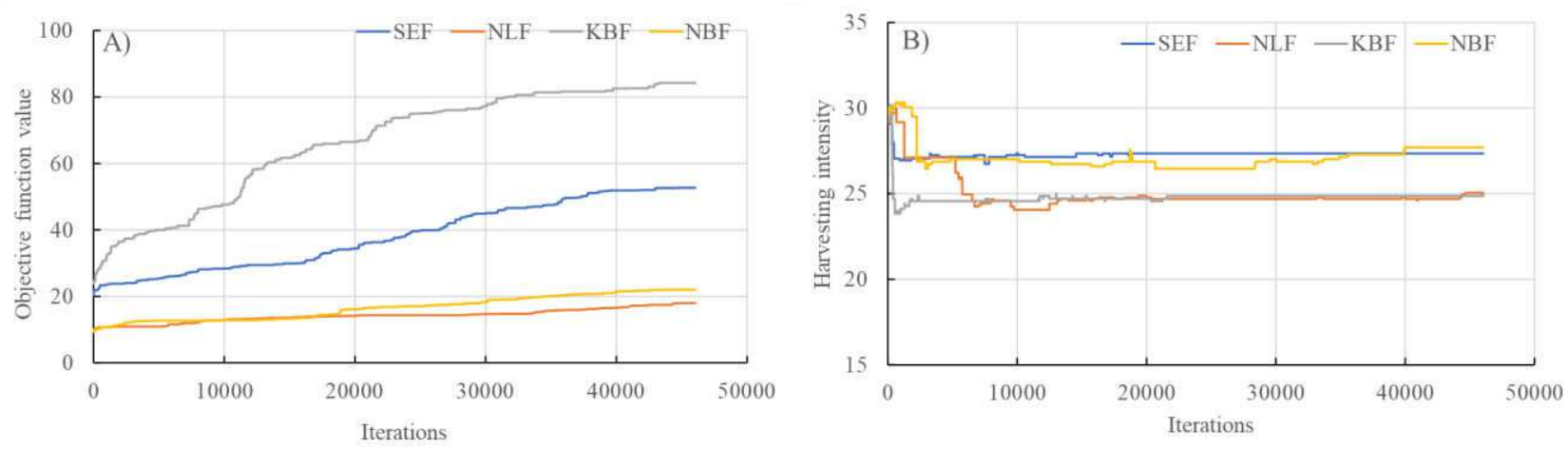

Figure 3 Developments of objective function values (left)c and harvest intensity (right) of the four case study forests in northeast

$$
\text { pine broadleaved forest. }
$$

The neighborhood-based structural characteristics for each of the tested forests before- and after-thinning indicate that parameter $M$ seemed to be the most important contributors to the objective function values for NBF forests (Table 3), while parameter $C$ for the other three forests (i.e., NLF, SEF and KBF). The initial values of $M$ for SEF and KBF were as large as 0.7036 and 0.7629 , which both belong to the category of high mixture, however the mingling degrees were quite lower for NLF (0.2789) and NBF (0.1105). Tree-level thinning improved the mean $M$-values by approximately $16.89 \%$ of NLF, $44.89 \%$ of NBF, $11.90 \%$ of SEF and $12.07 \%$ of KBF, respectively. The mean values of $C$ were decreased significantly from 0.7150 to 0.5329 of NLF (25.47\%), and from 0.4771 to 0.3494 of $\operatorname{NBF}$ (26.77\%), and from 0.8638 to 0.6659 of SEF (22.91\%), and from 0.8357 to 0.6670 of KBF (20.19\%), respectively, if the optimal harvest plans were implemented. The amounts of optimization on the $U$ were quite small when compared that with parameter $M$ and $C$, in which the decreases were only about $0.49 \%$ to $1.33 \%$. The effects of optimization on parameter $W$ highly depended on the forests, where the differences between the values of $W$ after thinning and an expect value (namely 0.5$)$ were decreased slightly for NBF $(0.4720$ vs 0.4818 , in a term of before- vs after- thinning) and KBF (0.5070 vs 0.4976$)$, while were increased for NLF 

were adjusted from a regular to a random distribution.

Table 3 Results of stand neighborhood-based structure optimization for the four tested forests in northeast China

\begin{tabular}{|c|c|c|c|c|c|c|c|c|}
\hline \multirow{4}{*}{ Variables } & \multirow{2}{*}{\multicolumn{2}{|c|}{ Natural larch forest }} & & & & & & \\
\hline & & & \multicolumn{2}{|c|}{ Natural birch forest } & \multirow{2}{*}{\multicolumn{2}{|c|}{ forest }} & & \\
\hline & & & & & & & \multicolumn{2}{|c|}{$\mathrm{KBF}$} \\
\hline & Before & After & Before & After & Before & After & Before & After \\
\hline Number of diameter class & 14 & 14 & 16 & 16 & 24 & 24 & 19 & 19 \\
\hline Number of tree species & 4 & 4 & 5 & 5 & 14 & 14 & 14 & 14 \\
\hline$Q$-value of diameter distribution & 1.57 & 1.54 & 1.46 & 1.44 & 1.31 & 1.29 & 1.23 & 1.21 \\
\hline Mingling index $M$ & 0.2789 & 0.3260 & 0.1105 & 0.1601 & 0.7036 & 0.7873 & 0.7629 & 0.8550 \\
\hline Diameter dominance index $U$ & 0.4886 & 0.4821 & 0.4855 & 0.4828 & 0.4890 & 0.4859 & 0.4676 & 0.4653 \\
\hline Uniform angle index $W$ & 0.4904 & 0.4874 & 0.4720 & 0.4818 & 0.5107 & 0.4856 & 0.5070 & 0.4976 \\
\hline Crowdedness index $C$ & 0.7150 & 0.5329 & 0.4771 & 0.3494 & 0.8638 & 0.6659 & 0.8357 & 0.6670 \\
\hline Harvest intensity of trees $\%$ & 24.96 & & 27.70 & & 27.35 & & 24.85 & \\
\hline Harvest intensity of volume $\%$ & 24.49 & & 26.04 & & 22.16 & & 22.23 & \\
\hline Objective function value & 10.0937 & 18.0044 & 9.3853 & 22.0518 & 20.5262 & 52.6898 & 23.8706 & 84.2523 \\
\hline
\end{tabular}
distribution were all not well-marked as expected (Table 3). However, slight decreases in the $Q$-values were 
observed for all of the four forests, indicating the increases on the proportion of smaller trees, yet all forests still had $Q$-values in reasonable ranges for natural uneven-aged forests. The assigned harvests mainly focused on the trees with DBH less than $15 \mathrm{~cm}$, namely $76.38 \%$ of NBF, and $61.31 \%$ of KBF, and $88.49 \%$ of NLF, and $74.35 \%$ of SEF, respectively (Figure 4).
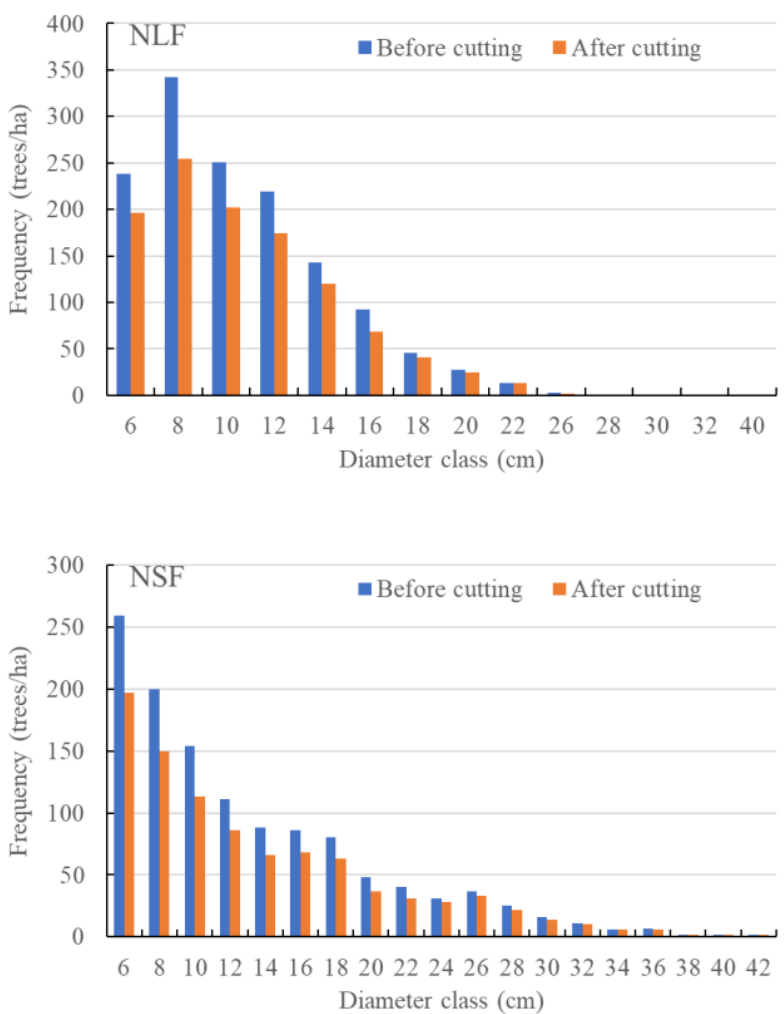
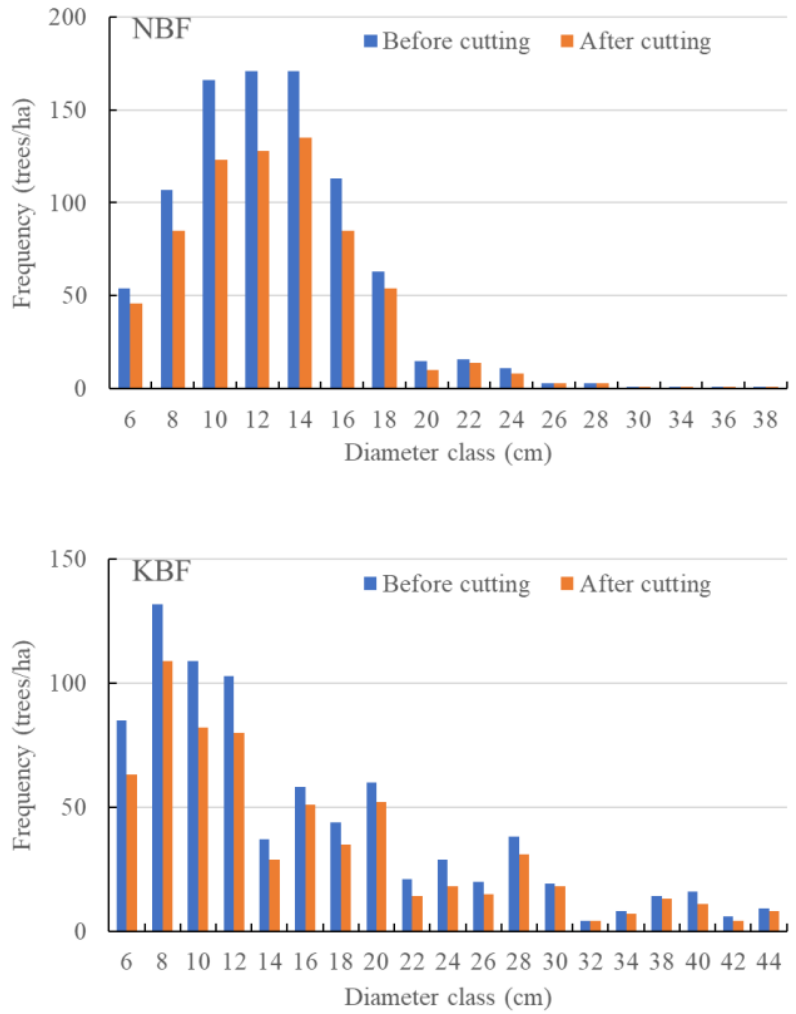

Figure 4 Distribution of diameter class for the four tested forests in northeast China, where SEF is the second growth forest, NLF is the natural larch forest, NBF is the natural birch forest and KBF is the Korean pine broadleaved forest.

Spatial distribution of the assigned harvest trees of optimal solutions for the four forests illustrates that the assigned harvest trees usually had the same tree species and dense crowdedness degrees within the structural units (Figure 5). The statistical results indicated that the assigned harvest trees mainly focused on B. platyphylla (97\%) of NBF, while L. gmelinii were dominated the harvest trees of NLF (80\%). However, the distribution on tree 

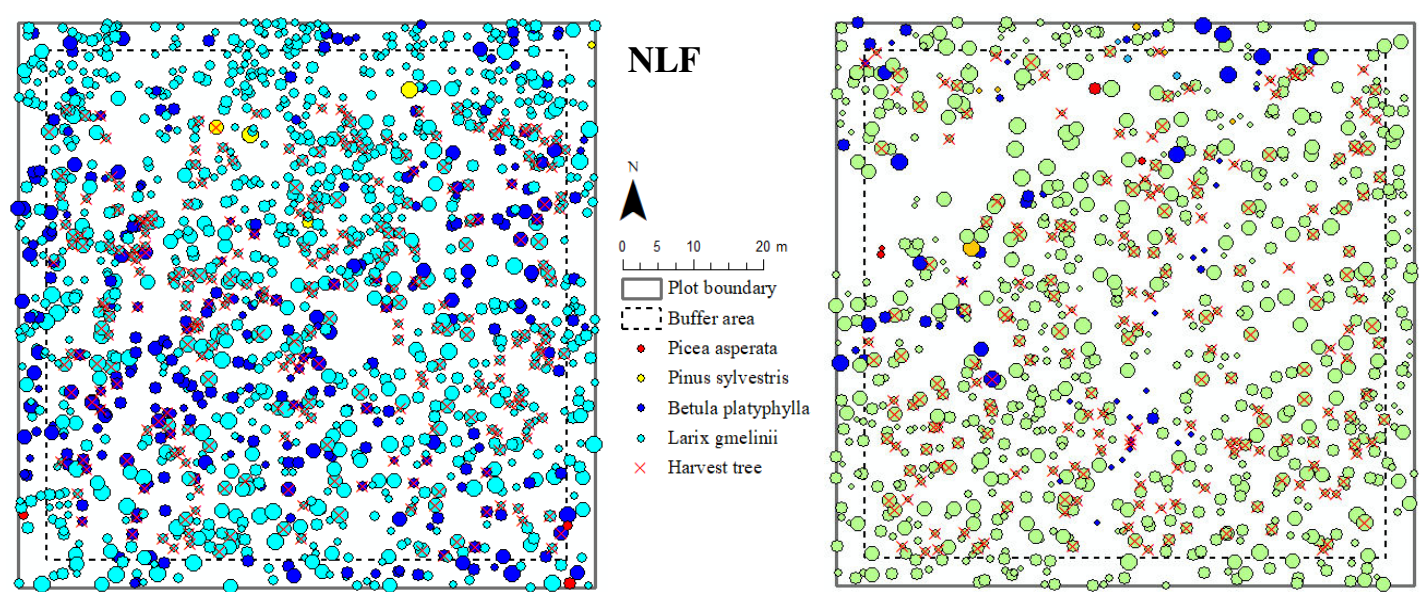

NBF
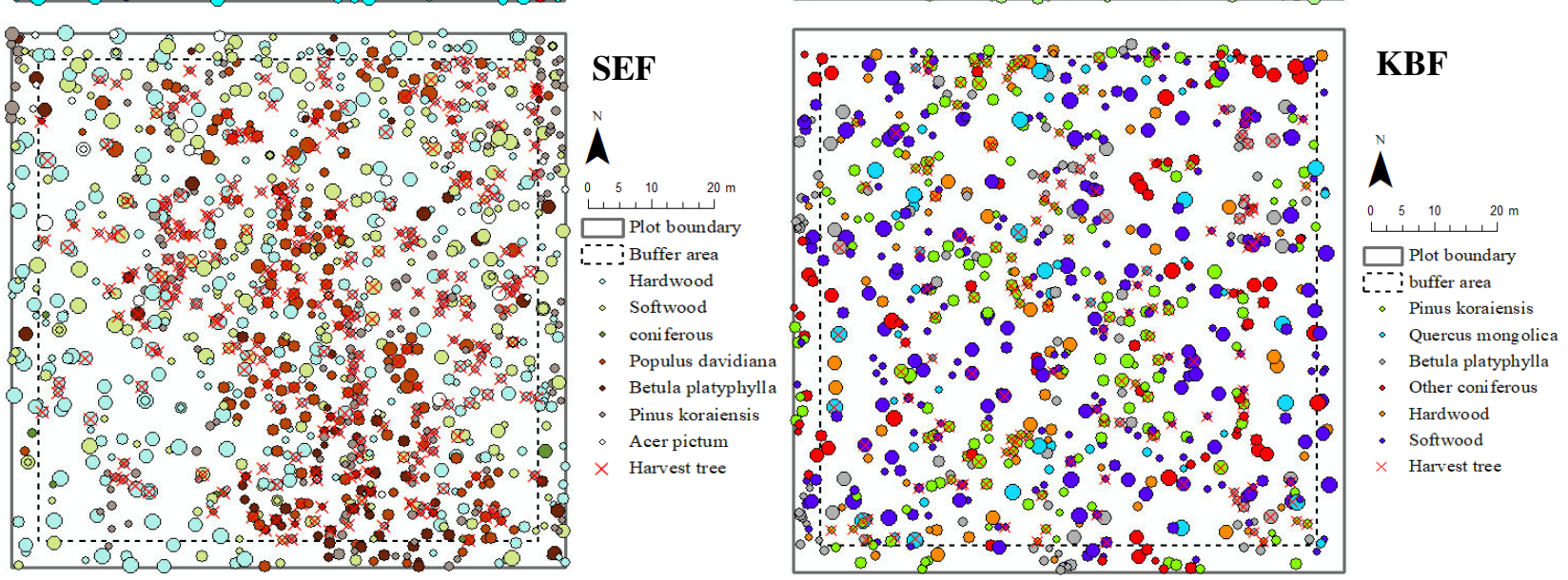

Figure 5 Spatial distribution of the assigned harvest tree for the four tested forests in northeast China, where SEF is the second

\section{Discussion}


healthy and stable forest (Hui and Gadow, 2003; Pastorella and Paletto, 2013). However, most management operations across the world are usually based only on the traditional forest management theory (e.g., normal forests, financial optimization) which focus on regulating stand density, diameter distributions, and species composition, while failing to consider the positive effects of stand spatial structure on the growth and stability of forests. Although these traditional techniques can significantly increase the growth space and decrease the competition status of remaining trees (Canellas et al., 2004; Forrester et al., 2013), the responses of stand spatial structural to management operations are always passive. This may be the main reason why the effects of traditional management techniques may not be as good as anticipated. Meanwhile, according to systems theory, reasonable stand structure is an important basis for ensuring continuous returns of various ecological benefits. Our approach, in which the potential harvest trees were selected in order to optimize stand spatial structure from four different aspects concurrently, perhaps better reflects the realistic forest management outcomes from a landowner's point of view. The results also highlight that spatially explicit tree-level harvest planning can be valuable for managing natural, mixed, uneven-aged forests.

As a healthy and stable forest, natural forests are usually recognized as ideal templates for uneven-aged forest management. Thus, the optimization principles presented in this study built upon the knowledge provided by previous studies regarding the structural characteristics of uneven-aged forests (Bettinger and Tang 2015, Li et al., 2017, Dong et al., 2020). The developments of horizontal distribution patterns are usually shifted from aggregation to random with forest succession, maintaining the random pattern consistently if the forest community could reach its climax status. Our results indicated that the trees grown in NLF, SEF and KBF stands were all randomly distributed (Table 3), which were perfect in line with the results of Wan et al. (2019) and Dong et al. (2020); however, slight aggregated distribution was observed for NBF, mainly due to a certain percentage of trees were sprouting regimentation in the stands. Regardless of the distribution pattern, the tree-level harvest 
optimization could produce significant randomly distributions immediately. The differences between the value of $W$ after thinning and the expected value (namely 0.5 ) were decreased for NBF and KBF, indicating the horizonal distribution of trees would become much more random, while slight increases were observed for NLF and SEF. These results were all perfectly in line with the scope of structure-based forest management strategies (Hui and Gadow, 2003).

The diversities of species and neighborhood-based structural are both quite important for understanding the ecological processes and implementing sustainable forest management. The spatially explicit species mingling index $(M)$ belongs to the typical local diversity measures, which is inversely proportional to the relative tree density of each tree species within a structural unit, yet may decrease significantly with increases in homogeneity in the stands (Bettinger and Tang, 2015; Pommerening and Uria-Diez, 2017). Thus, the indicator of $M$ was employed as an important proxy of diversity across the entire stand to instead of the traditional diversity index, such as Shannon-Wiener index (Shannon and Weaver, 1949). The outcome of this study indicated that the values of $M$ increased significantly with increases in species richness, and their relative abundance, namely ranging from 0.11 of NBF to 0.76 of KBF (Table 3), represented significant variations across a latitude gradient. However, we further found that improving the value of $M$ was completely opposite with the increases of species richness, in which the largest increases were observed for the simplest stands (NBP; 44.87\%), while the increases were quite smaller for the two much more complex stands, namely $11.90 \%$ of SEF and $12.07 \%$ of KBF. Lyu et al. (2020) have once stated that increasing the values of $M$ by $10 \%$ for natural L. gmelinii forest could enlarge the DBH of individual tree significantly by an average of $4.71 \mathrm{~cm}$. Thus, improving species mingling through tree-level harvest such as this may be conducive to tree growth.

Reducing the competition pressure of remaining trees is another important concern in forest management. In this study, two commonly used competition-related measures were considered. The first parameter $(D)$ is related 
to the diametric differentiation of trees, while the second parameter $(C)$ mainly refers to the forest coverage. From the results, the differences on the mean values of $D$ among different forests, as well as among different thinning scenarios for each forest (namely $0.49 \%-1.33 \%$ ), were all quite difficult to distinguish (Table 3). Thus, we concluded that the parameter $D$ seemed to be not very suitable to describe the diameter differentiations at stand-level, even so the effects of parameter $D$ at tree-level remains unquestioned. To overcome this imperfection, some other indicators of size dominances such as height dominance, diameter differentiation, diameter correlation index and crown layer differentiation (Davies and Pommerening, 2008) could be further verified in the near future. However, the sensitiveness of parameter $C$ was quite satisfactory when was employed to evaluate the crowdedness among different forests and thinning scenarios. The decreases on the mean values of $C$ after thinning were as large as $20.19 \%-26.77 \%$, implying the lighting areas on the crown could be increased significantly. As emphasize here, the commonly used Hegyi competition index (Hegyi, 1974) was not considered in this analysis, mainly because it needs detailed distance information, which is a time- and cost-consuming survey measure. Although our case studies were implemented on a fully mapped stands, the four neighborhood-based parameters are quite easy to be visual checked in the forests, as shown in Figure 1.

The present study advances the management of natural forests from the perspective of optimizing stand neighborhood-based structural, however some limitations still existed in our tree-level harvest planning. The first, and perhaps the most important, was that some important nonspatial features were not fully considered in the optimization process. For instance, the $Q$-values of diameter distribution were only optimized as a constraint rather than objective, and the intervals of $Q$-value were also only determined from experience perspectives (e.g., Kang, 2010). These may be the main reasons for the poor distributions of diameter after thinning. The second mainly refers to the operability for field personnel when the optimization approach is extended to a larger forest area, which can be approached from two different aspects. On one side, numerous training processes as 
398

implemented in this study can provide some prior knowledge for tree marking decisions which are helpful to help improve understanding of field personnel on the management of forests. On the other side, the relatively accurate detection capability of LiDAR on identifying the locations of trees and their marks (e.g., HT, CW) may further accelerate the application of tree-level harvest planning approach in natural forests (Packalen et al., 2020; Pascual, 2021). The last, but not least, is that the future outcomes of the logged stands based on the benefits of growth, economic, and environmental concerns are still not clear, thus combining our tree-level harvest optimization process and forest growth and yield models that are sensitive to the neighborhood-based structural parameters is imperative.

\section{Conclusions}

The proposed tree-level harvest planning approach, considering four different aspects of neighborhood-based structural and recognizing other operational constraints, could be used effectively to manage natural mixed uneven-aged forests. The applications of this approach in the four tested forests indicated that the objective function values could be significantly improved by approximately $78.33 \%$ of NLF, and $134.96 \%$ of NBF, and $156.70 \%$ of SEF and $252.95 \%$, respectively. The corresponding harvest intensities of removing volumes varied between $22.16 \%(\mathrm{SEF})$ and $26.07 \%(\mathrm{NBF})$. Both species mingling and crowdedness have the highest priority to be adjusted in structure-based forest management.

\section{Acknowledgements}

Not applicable.

\section{Authors' contributions}

ZL conceived and designed the study; LD and PB analyzed and interpreted the data and wrote the primary draft. All authors read and approved the final manuscript. 


\section{Funding}

This work was supported by the National Natural Science Foundation of China (Grant number 3217140069

421 the Fundamental Research Funds for the Central University [grant number 2572019BA07]; and the Heilongjiang

422 Touyan Innovation Team Program (Technology Development Team for High-efficient Silviculture of Forest

423 Resources).

\section{Availability of data and materials}

425 The datasets used during the current study are available from the corresponding author on reasonable request.

426 Declarations

Ethics approval and consent to participate

428 Not applicable.

\section{Consent for publication}

$430 \quad$ Not applicable.

\section{Competing interests}

432 The authors declare that they have no competing interests.

\section{Author details}

$434{ }^{1}$ Key laboratory of Sustainable Forest Ecosystem Management-Ministry of Education, College of Forestry,

435 Northeast Forestry University, Harbin 150040, China; ${ }^{2}$ Warnell School of Forestry and Natural Resources, 436 University of Georgia, Athens 30602 GA, USA. 
simulation. Royal College of Forestry, Stockholm.

Hui GY, Gadow Kv (2003) Quantitative analysis of forest spatial structure. Beijing: Science and Technology Press.

Hui G, Li L, Zhao Z, Dang P (2007) Comparison of methods in analysis of the tree spatial distribution pattern. Acta Ecologica Sinica 27(11): 4717-4728.

Hui G, Gadow Kv, Hu Y, Chen B (2004) Characterizing forest spatial distribution pattern with the mean value of uniform angle index. Acta Ecologica Sinica 24(6): 1225-1229. (In Chinese)

Kang XG (2010) Forest Management ( $4^{\text {th }}$ edition). Beijing: China Forestry Publishing House (in Chinese).

Kirkpatrick S, Gelatt Jr CD, Vecchi MP (1983) Optimization by simulated annealing. Science 220(4598): 671-680.

Li Y, Ye S, Hui G, Yanbu H, Zhonghua Z (2014) Spatial structure of timber harvested according to structure-based forest management. Forest Ecology and Management, 322: 106-116.

Li Y, Hui G, Wang H, Zhang G, Ye S (2017) Selection priority for harvested trees according to stand structural indices. iForest 10(3): 561-566.

LV YH, Yi LQ, Wang RL, Liu ZG, Dong LB (2020) Diameter growth model using spatial structure parameters of natural Larix gmelinii stand in Daxing'anling Mountains, Northeast China. Forest Research 34(2): 81-91

MacDonald GB, Cherry ML, Thompson DJ (2004) Effect of harvest intensity on development of natural regeneration and shrubs in an Ontario boreal mixedwood stand. Forest Ecology and Management 189: 207-222.

Martín-Fernández S, García-Abril A (2005) Optimisation of spatial allocation of forestry activities within a forest stand. Computers and Electronics in Agriculture 49(1): 159-174.

Metropolis N., Rosenbluth AW, Rosenbluth MN, Teller AH, Teller E (1953) Equation of state calculations by fast computing machines. Journal of Chemical Physics 21(6): 1087-1092.

Newton PF (2012) A decision-support system for forest density management within upland black spruce stand-types. Environmental Modelling \& Software 35: 171-187. 
http://www.forestry.gov.cn/main/446/content-892764.html [Feb-09-2021]

Wan P, Zhang G, Wang H, Zhoa Z, Hu Y, Zhang G, Hui G, Liu W (2019) Impacts of different forest management methods on

507 the stand spatial structure of a natural Quercus aliena var. acuteserrata forest in Xiaolongshan, China. Ecological Informatics

508 50:86-94.

Wang H, Zhang G, Hui G, Li Y, Hu Y, Zhao Z (2016) The influence of sampling unit size and spatial arrangement patterns on

neighborhood-based spatial structure analyses of forest stands. Forest Systems 25(1): e056. 